\title{
LA PERSPECTIVA DE GÉNERO EN LA FORMACIÓN INICIAL DEL PROFESORADO DE EDUCACIÓN INFANTIL. NOTAS SOBRE UNA INVESTIGACIÓN REALIZADA ENTRE EL ALUMNADO DEL GRADO DE MAGISTERIO DE EDUCACIÓN INFANTIL DE LA UNIVERSIDAD DE CANTABRIA (ESPAÑA)
}

Marta García Lastra*

\section{Resumen}

A pesar del favorable contexto legal vivido en España durante las primeras décadas del Siglo XXI para el tratamiento de la perspectiva de género en todos los niveles educativos, la realidad nos hace ver cómo ésta no ha sido abordada adecuadamente.

Se presentan en este artículo los resultados de una investigación realizada entre el alumnado del $1^{\circ}$ curso de Magisterio de Educación Infantil de la Universidad de Cantabria (España) para conocer su opinión sobre la situación de mujeres y hombres en la sociedad, su conocimiento hacia la coeducación, el papel que la escuela debe jugar en la búsqueda de la igualdad de género además de su valoración sobre la inclusión de estas cuestiones en el Grado. El trabajo está basado en una encuesta y un grupo de discusión. Infantil.

Palabras clave: Perspectiva de género, coeducación, universidad, Grado Educación

\section{Resumo}

A perspetiva de género na formação inicial de docentes de educação infantil. Notas sobre uma investigação realizada com estudantes da licenciatura em educação infantil da Universidade de Cantábria (Espanha)

Apesar do contexto jurídico favorável vivido em Espanha durante as primeiras décadas do século XXI para a inclusão da perspetiva de género em todos os níveis educacionais, a realidade mostra que essa abordagem não é devidamente feita nos diferentes anos de escolaridade.

Discutem-se neste artigo os resultados de uma investigação realizada com alunos e alunas do $1 .^{\circ}$ curso da Licenciatura em Educação Infantil da Universidade de Cantábria (Espanha), a fim de apresentar os seus pontos de vista acerca da situação das mulheres e dos homens na sociedade, bem como descobrir o conhecimento que possuem sobre o que é a educação mista, qual o papel que a escola deve desempenhar na promoção da igualdade, além da perceção que têm da oportunidade de abordar estas questões no currículo do curso que frequentam. A recolha de dados foi feita através de um inquérito e de um grupo de discussão.

Palavras-chave: Perspectiva de género, coeducação, universidade, licenciatura em educação infantil.

Facutad de Educatión, Universidad de Cantabria, España.

Endereço eletrónico: garciama@unican.es

Dirección postal: Universidad de Cantabria, Facultad de Educación, Avenida de los Castros, s/n, 39005 Santander (España). 
Abstract

Gender perspective in initial training for early childhood education teachers: notes about a research carried out in the degree of early childhood education at the University of Cantabria (Spain)

In spite of the favourable legal context in Spain during the first decades of the $21^{\text {st }}$ century for the gender mainstreaming perspective at all educational levels, reality shows that it has not been addressed adequately.

In this paper, we present the results of a research carried out in the 1st course of the Degree in Early Childhood Education at the University of Cantabria (Spain), in order to know students' opinions on the situation of women and men in society, their perceptions about coeducation programmes, the role school should play in the pursuit of equality and students' vision about the opportunity for incorporating this subject in their degree programme. The research is based on a survey and a focus group.

Keywords: Perspective, coeducation, university, Degree in Early Childhood.

\section{Introducción: un contexto favorable y una oportunidad perdida}

La primera década del Siglo XXI bien puede ser considerada como un momento especialmente favorable para la inclusión de la perspectiva de género en el mundo de la educación en España. Junto a las diversas leyes educativas de todos los niveles promulgadas en este decenio que incorporaron la promoción de igualdad entre mujeres y hombres en su articulado, otras como la Ley de Medidas de Protección Integral contra la Violencia de Género (Ley Orgánica 1/2004, de 28 de diciembre), la Ley para la igualdad efectiva de hombres y mujeres (Ley 3/2007, de 22 de marzo) y, en 2011, la Ley de la Ciencia, la Tecnología y la Innovación (Ley 14/2011, de 1 de junio), incluyeron propuestas de actuación en el ámbito educativo.

Además, en el contexto universitario, al amparo del artículo 45.2 de la Ley de Igualdad de $2007^{1}$ se promovieron planes de igualdad entre mujeres y hombres en las empresas que, en el caso de la Universidad de Cantabria (UC), echó a andar en el año 2009. El plan fue elaborado por la Comisión Transversal de Igualdad, órgano consultivo y asesor creado un año antes y en el que encuentran representación todos los sectores de la comunidad universitaria - Personal Docente e Investigador (PDI), Personal de Administración y Servicios (PAS), estudiantado, sindicatos y gerencia. Dicho plan recoge, entre otras, una serie de recomendaciones en materia de igualdad, como la promoción del conocimiento de la Ley de Igualdad y sus implicaciones; la introducción de la variable de sexo en las estadísticas u otro material utilizado en clase; la inclusión de aspectos relacionados con la perspectiva de género en el programa del curso o la visibilización de la contribución

Según el artículo 45.2 de la citada Ley: «En el caso de las empresas de más de doscientos cincuenta trabajadores, las medidas de igualdad a que se refiere el apartado anterior deberán dirigirse a la elaboración y aplicación de un plan de igualdad, con el alcance y contenido establecidos en este capítulo, que deberá ser asimismo objeto de negociación en la forma que se determine en la legislación.» 
al conocimiento realizada por las mujeres en cada disciplina (García Lastra y Díaz Díaz 2013).

Este contexto legal favorable corría parejo a un cambio en el escenario universitario protagonizado por la llegada del Espacio Europeo de Educación Superior (EEES), cuyas normativas a la hora de confeccionar los programas de los nuevos grados y postgrados parecían respirar los mismos aires: así, el Real Decreto 1393/2007, de 29 de octubre, ${ }^{2}$ sobre la elaboración de los nuevos títulos, establecía en su artículo 3.5.a la inclusión de enseñanzas relacionadas con la igualdad entre mujeres y hombres. Una situación recogida por los diferentes seminarios, grupos de investigación e institutos universitarios de Estudios de las Mujeres y del Género que en su «Declaración por la plena integración de los estudios de las mujeres, feministas y de género en el EEES» demandaban, entre otras actuaciones, «la inclusión de asignaturas troncales y obligatorias adaptadas a las diferentes titulaciones universitarias con objeto de introducir la aportación de los estudios de género en las distintas disciplinas, teniendo en cuenta las que ya se imparten como optativas o de libre elección» (citado por Segura Graíño 2010, 75).

Sin embargo, como ya se ha señalado en otras investigaciones (Romero Díaz y Abril Morales 2008, Anguita y Torrego 2009, Ballarín 2013, Donoso-Vázquez y Velasco-Martínez 2013, Verge Mestre y Cabruja Ubach 2017, entre otros), la puesta en marcha de los nuevos planes de estudio bien puede ser considerada una oportunidad perdida para la incorporación de la perspectiva de género. Esta perspectiva, entendida, siguiendo a Donoso-Vázquez y Velasco-Martínez, como el «posicionamiento crítico para el análisis de la dimensión crítica/evaluativa de la normatividad heterosexual y patriarcal que sustenta todo el sistema social y en el que el género es un principio de jerarquización de espacios, recursos materiales, económicos, públicos e ideológicos» $(2013,75)$, supone la eliminación del sexismo y la búsqueda de la igualdad entre mujeres y hombres.

Una perspectiva que toma carta de naturaleza en los Estudios de las Mujeres y del Género o feminismo académico, los cuales nacidos bajo la idea de la promoción de nuevos saberes asociados a los secularmente femeninos y el desarrollo de una nueva perspectiva alejada del orden androcéntrico, han promovido desde sus orígenes un proceso de transformación social para la consecución de un nuevo tipo de sociedad en la que el patriarcado desapareciera como sistema social de poder (Coyner 1991, Dickinson 2005).

De este modo, los conocimientos desarrollados desde los Estudios de las Mujeres se convierten, junto a las personas implicadas en ellos, en el marco de referencia fundamental para el desarrollo de asignaturas en materia de género o la introducción de este tema en las ya existentes. Creo que la formación específica en esta cuestión, al igual que es demandada para la impartición de cualquier otro tipo de conocimiento, debe ser considerada imprescindible, partiendo además de la convicción de la responsabilidad de la institución universitaria en la extensión

$2 \quad$ Última modificación: 3 de junio de 2016. 
de la igualdad de oportunidades, un espacio en el que el profesorado debe jugar un papel fundamental en su transmisión (López-Francés et al. 2016). Considero, siguiendo a Ballarín (2013), que no sólo hace falta una actitud proclive para su tratamiento en las aulas universitarias, sino también conocimiento científico, de otro modo, parafraseando a esta autora, se quedaría en la «conciencia sin ciencia».

Estos Estudios, aun habiendo llegado con retraso al contexto español respecto a otros países europeos (Woptipka et al. 2007), comenzaron a ocupar a partir de los años 1980 un espacio en las universidades españolas que se afianzó una década después (García Lastra 2010a). De este modo, su consolidación en la universidad española (medida a través de la creación de institutos, seminarios, la aparición de publicaciones, el desarrollo de investigaciones por el PDI, etc.) y los conocimientos adquiridos por el profesorado vinculado a esta tradición (Ballarín 2013, Vizcarra et al. 2015), facilitaba la implantación de asignaturas no sólo optativas (entre las que se pueden encontrar algún ejemplos), sino básicas u obligatorias en los nuevos grados. Y, especialmente, la perdida de esta oportunidad debe hacerse notar en los estudios desarrollados en las facultades de educación y de formación del profesorado, donde a pesar de la denuncia de algunos estudios como el realizado por el Instituto de la Mujer (2004) que alertaban sobre la escasa formación en materia de género por parte del profesorado, la situación no fue distinta a la vivida en otros centros universitarios a la hora de confeccionar sus planes de estudio.

\section{El papel de la escuela en la búsqueda de la igualdad entre mujeres y hom- bres}

No se puede dejar de reivindicar el papel de la escuela, y de sus docentes, en la deconstrucción de roles de género, de conductas sexistas que siguen reproduciéndose en nuestra sociedad, que se manifiestan desde las actitudes más o menos sutiles que nos acompañan diariamente, y que siguen menospreciando, ridiculizando o minusvalorando a las mujeres, hasta las cruentos episodios de violencia de género. Situaciones que, a pesar de los innegables avances en materia de igualdad a nivel formal, parecen perpetuarse entre una parte importante de la gente más joven: a modo de ejemplo, en España, el 30\% de los chicos de entre 12 y 24 afirma que «Cuando la mujer es agredida por su marido, algo habrá hecho ella para provocarlo», un porcentaje que desciende hasta el $11 \%$ en el caso de las chicas $^{3}$ (Megías y Ballesteros 2015).

Como ya se ha dicho en otras ocasiones (Simón 2010, Fernández Enguita 2016), la escuela bien puede ser considerada una de las instituciones sociales menos discriminatoria por razón de género, especialmente si se compara con otros contextos especialmente hostiles para las mujeres como el mundo del trabajo e

El porcentaje corresponde a la suma de las respuestas «Algo de acuerdo», «Bastante de acuerdo $\mathrm{y}$ «Muy de acuerdo». 
incluso la familia. Si bien, a pesar de esta situación, y del importante camino recorrido por las mujeres en su escolarización, desde su exclusión hasta la llegada a una escuela mixta (Calvo et al. 2011), la actual institución escolar continúa reproduciendo en su seno códigos de género secularmente aceptados que perpetúan el sexismo y que nos llevan a negar la supuesta neutralidad de la escuela mixta y a seguir reivindicando la llegada de la escuela coeducativa. De este modo, se sigue encontrando con desigualdades de trato, actitudes y expectativas del profesorado; con diferentes usos de los espacios por parte del alumnado; con conocimientos androcéntricos trasmitidos a través del currículo y por ende a la no consideración de saberes tradicionalmente femeninos en él; con libros de texto en los que la presencia de las mujeres sigue siendo insignificante y reducida a determinados papeles y espacios; con un uso generalizado del lenguaje sexista o con la desigual y pertinaz presencia de hombres y mujeres en los puestos de responsabilidad del mundo educativo (García Lastra 2010b; López Navajas 2014; Subirats 2016).

Confiar en el papel transformador de la escuela ante estas situaciones implica asumir la coeducación como modelo educativo y la perspectiva de género como metodología de éste. Una perspectiva que, como recuerda Rebollo (2013), debe ser considerada un agente para la innovación y el cambio educativo. Sin embargo, si bien durante las décadas de los 1980 y 1990 la etapa de Educación Infantil, Primaria y Secundaria fue testigo de la puesta en marcha de importante número de proyectos en materia coeducativa, los años posteriores han visto cómo el interés por el tema ha ido descendiendo (Romero Díaz y Abril Morales 2008). De este modo, la realidad nos muestra cómo, a pesar de que la formación para la ciudadanía ha sido considerada una de las funciones esenciales de la escuela (Fernández Enguita 2016), ésta ha soslayado en numerosas ocasiones su responsabilidad a la hora de poner en marcha medidas en pro de la igualdad. Reticencias que en ocasiones han partido desde los propios centros educativos, desde sus claustros, en los cuales bajo la suposición de que la igualdad es algo conseguido, se ha dejado de lado su compromiso de intervención a través de la práctica docente. Como afirman Rebollo et al (2011) en su investigación, las principales dificultades destacadas por los responsables, o expertos, en coeducación en los centros educativos a la hora de desarrollar lo que denominan la «cultura de género» es la baja importancia concedida al clima de género en la escuela (estado de opinión de la comunidad educativa sobre la coeducación y la igualdad), la falta de formación del profesorado y la falta de aplicabilidad de las políticas de género (la continuidad, transparencia y aplicabilidad de las acciones relacionadas con la igualdad).

Como insiste Simón (2010, 129-130), si bien el principio de la igualdad se ha instalado, no ha pasado lo mismo con su praxis, de tal modo que la escuela debe promover esta práctica, «adaptando sus modos y contenidos a una intervención decididamente coeducativa, educación con enfoque de género y no sexista, que contenga bastantes componentes hasta ahora ausentes o con implantación muy débil y dispersa» (2010, 129-130). En este sentido, la puesta en marcha de una escuela coeducativa debe de ser contemplada tanto como un fin como un medio. En 
el primero de los casos, la coeducación, formando parte de la idea de escuela inclusiva, constituirá el último eslabón en la periodización del proceso de la relación de las mujeres con el sistema educativo antes referido De otro, este tipo de escuela conseguirá terminar con situaciones que, presentes en el mundo escolar, continúan perpetuando los estereotipos de género y promoviendo las relaciones desiguales.

\section{La investigación realizada}

El trabajo que aquí se presenta se plantea como una indagación de los presupuestos más arriba expuestos en un contexto determinado, esto es, entre el alumnado matriculado en el curso 2015-2016 en la asignatura «Sociedad, Cultura y Educación» impartida en el primer curso del Grado de Magisterio de la Universidad de Cantabria. Esta asignatura se desarrolla durante el primer cuatrimestre del curso e incluye entre sus bloques temáticos el tema «Desigualdades sociales, desigualdades educativas» dentro del cual se abordan las desigualdades de género. El alumnado se divide en tres grupos, dos de turno de mañana y uno de tarde.

Los objetivos de la investigación se centraran en analizar su conocimiento sobre la coeducación y acciones educativas en materia de género; su opinión sobre la situación de mujeres y hombres en la sociedad; el papel que la escuela debe jugar en la búsqueda de la igualdad de géneros además de su valoración sobre la inclusión de la formación en género en el Grado.

\section{Metodología e instrumentos de recogida de datos}

Para la realización del trabajo, se ha combinado metodología cuantitativa y cualitativa. Entiendo, siguiendo a Ruiz Olabuénaga (2003), que esta «triangulación» de metodologías enriquece el análisis final mediante una estrategia de complementariedad. Según este mismo autor, esta alternativa metodológica puede realizarse de diversas maneras, una de ellas utilizando la perspectiva cualitativa a posteriori de la cuantitativa, opción elegida para este trabajo.

En concreto, se ha llevado a cabo una encuesta y un grupo de discusión, técnicas que pasaré a detallar a continuación. De este modo, «se pondrá voz» (a través de la encuesta) y «se dará voz» (a partir del grupo de discusión) a nuestro alumnado.

Para la realización de la encuesta se elaboró un cuestionario ad hoc tras la revisión de investigaciones recientes sobre el tema (Romero Díaz y Abril Morales 2008; Fernández García y Piedra 2010; Suberviola Ovejas y Goicoechea 2010; López-Francés et al. 2016), creándose una batería de veintiún preguntas organizadas en varios apartados temáticos (además de preguntas sociodemográficas): conocimiento y significado de la coeducación; participación en programas de igualdad durante su etapa educativa previa a la universidad; papel de la escuela en la búsqueda de 
la igualdad entre mujeres y hombres; importancia de la formación en género en el Grado de Magisterio; grado de acuerdo sobre diversas cuestiones relacionadas con la igualdad entre géneros. En este último caso, se utilizó una escala tipo Lickert con valores de 5 (Muy de acuerdo) a 1 (Muy en desacuerdo). Para el resto del cuestionario, se combinó la formulación de preguntas cerradas (con respuestas dicotómicas o de elección múltiple) y abiertas (que permitían explicar alguna de las respuestas dadas).

Antes de la realización de la encuesta, se realizó una prueba piloto del cuestionario entre varios estudiantes de la titulación que sirvió para comprobar su inteligibilidad. Fueron modificadas dos preguntas al ser consideradas, a juicio del estudiantado, confusas en su formulación.

El cuestionario fue presentado y explicado por el profesorado de la asignatura en cada uno de los grupos durante los meses de noviembre y diciembre de 2015. Fue cumplimentado de manera voluntaria durante las horas de clase y sólo dos personas de las presentes en el aula durante su realización decidieron no responderlo. En total, 98 estudiantes respondieron a la encuesta (de un total de 116 matriculados).

Tras la grabación de los datos, se calcularon estadísticos descriptivos: frecuencias además de medias en el caso de las preguntas tipo Lickert. Las respuestas de las preguntas abiertas fueron categorizadas en un sistema de códigos emergentes.

El grupo de discusión fue realizado en febrero de 2016 tras el análisis de los resultados obtenidos a través de la encuesta. Su ubicación temporal tras este análisis permitió profundizar en algunos de los resultados obtenidos en el análisis cuantitativo. El grupo, elegido a través de un muestro intencional guiado por criterios de sexo, edad y estudios previos a la entrada al Grado, estuvo formado por seis estudiantes de la asignatura: cinco mujeres y un hombre; cuatro con edades entre 17 y 20 años, una de entre 21 y 25 y otra alumna con más de 25 años. Antes de su ingreso en el Grado, dos habían cursado el Ciclo Formativo de Técnico/a en Educación Infantil ${ }^{4}$ y habían realizado las prácticas de estas enseñanzas en escuelas de infantil ${ }^{5}$. El número de miembros, duración, organización..., estuvo guiado por los criterios de Gil-Flores (1992).

El guion del grupo de discusión, partiendo, como se dice, del análisis de los datos cuantitativos, se articuló en torno a los mismos ejes temáticos utilizados en el cuestionario, si bien introduciendo cuestiones que permitieran profundizar en algunos de los resultados obtenidos. Tras su transcripción y lectura, se extrajeron las principales conclusiones de los discursos del grupo para cada uno de estos ejes.

$4 \quad$ Me refiero a la titulación «Técnico/a superior en Educación Infantil», una enseñanza de carácter profesional que faculta para trabajar como educador/a en el primer ciclo de Educación Infantil (0-3 años) bajo la supervisión de un/a maestro/a.

$5 \quad$ A lo largo del artículo, los discursos transcritos aparecerán entrecomillados. 


\section{Resultados}

Atendiendo al perfil del alumnado que respondió a la encuesta, se debe señalar que $88 \%$ eran mujeres y que la mayor parte del estudiantado, el 55\%, tenía ente 17 y 20 años, un 38\% entre 21 y 25 y el $7 \%$ restante más de 25 .

Del análisis de los datos cuantitativos, la primera conclusión que se desprende es el escaso conocimiento del alumnado sobre el significado de la coeducación. En concreto, ante la pregunta ¿Sabes lo que significa el término coeducación?, un $29 \%$ admite no conocerlo y un $52 \%$ dice no tenerlo claro. Sólo el $18 \%$ afirma que sí, aunque, cuando es preguntado por su definición, se refiere a cuestiones que bien pueden hacer referencia a la escuela mixta y llevarnos a hablar de la confusión entre ambos términos. De este modo, educar para hombres y mujeres por igual o educar en igualdad de condiciones son algunas de las respuestas recogidas a partir de una pregunta abierta que pueden reflejar esta idea. Es significativo además que el alumnado que ha respondido sí conocer lo que significa la coeducación señale al entorno educativo (colegio, instituto o universidad) como el lugar donde lo ha aprendido: así, el 52,3\% habla de la escuela o el instituto y el 19\% de la universidad. El 14,3\% señala a la familia, el 9,5\% a los medios de comunicación y el 4,8\% a su grupo de amistades.

Esta situación bien puede estar relacionada con el escaso recuerdo sobre su participación en programas o proyectos coeducativos durante su etapa en la educación primaria o secundaria: preguntados/as por esta posibilidad (Durante tu paso por el colegio o instituto, ¿participaste en algún programa dirigido a trabajar la igualdad entre mujeres y hombres?), sólo un $17 \%$ recuerda haberlo hecho y un $25 \%$ si bien sabe que participó no puede concretar en qué consistía la actividad. No se debe olvidar que su paso por estas etapas educativas, dada la edad del alumnado encuestado, coincide con el momento de promulgación de la Ley de Igualdad y en el plano educativo de la Ley de Educación, quien en su Preámbulo afirma que:

Entre los fines de la educación se resaltan el pleno desarrollo de la personalidad y de las capacidades afectivas del alumnado, la formación en el respeto de los derechos y libertades fundamentales y de la igualdad efectiva de oportunidades entre hombres y mujeres. (Ley Orgánica 2/2006, 10)

Y, además, dedica la Disposición Adicional Vigesimoquinta al Fomento de la Igualdad entre hombres y mujeres en los centros escolares.

Entre el alumnado que recuerda haber participado destacan los que hablan del marco de una asignatura optativa denominada "Igualdad entre mujeres y hombres» durante la educación secundaria, siendo la violencia de género la temática que con mayor frecuencia se repite a la hora de acordarse de las actividades realizadas: elaboración de pancartas en contra que colocaron en el instituto de educación secundaria e incluso, en otro caso, llevaron a las puertas del Parlamento Regional, charla con mujeres que habían sido víctimas de esta violencia, etc. 
Casi la totalidad del alumnado encuestado $(98,9 \%)$, considera que la escuela debe trabajar en la búsqueda de la igualdad entre mujeres y hombres, encontrándonos sólo un $1 \%$ que afirma que no es necesario ya que ésta ya está conseguida. Cuando son preguntados, a través de una pregunta abierta, cómo debe realizarse este trabajo en el contexto escolar, la idea de actividades prácticas impregna la mayoría de las respuestas. De este modo, talleres, charlas, películas seguidas de coloquios, ejemplos reales..., aparecen como ideas fundamentales, pero no sólo para el alumnado, sino también para las familias, para que puedan presentar sus dudas, sus inquietudes, dado que a veces igual no dejan hacer a su hijo o hija algo por miedo a la sociedad, a lo que digan los vecinos.

Actividades que son además demandadas por el grupo de discusión desde la escuela infantil, dado que «es el lugar donde empezamos a conocernos, a vernos diferentes». Nos gustaría además señalar cómo en algunos casos se apunta a que éstas no deben ser actividades puntuales, «no dedicando única y exclusivamente un día a ello» e incluso reclamando una asignatura obligatoria dedicada a este tema.

Relacionado con esta idea, bien puede afirmarse que existe una demanda significativa hacia la inclusión de temas de igualdad en la formación inicial del profesorado: el $80 \%$ del alumnado consultado demanda este tipo de formación. Iniciarse en ella implica, no se lo olvide, comenzar a pensar en su práctica en el aula con perspectiva de género desde su formación inicial.

El grupo de discusión nos ofrece matices que permiten complementar los datos del cuestionario sobre esta cuestión. Sus miembros coinciden en la importancia de la consideración de la temática en el plan formativo de su titulación, «para que como docentes no nos pasen desapercibidas muchas cosas». Creen que «es importante que en el Grado se traten estos temas, una vez que ves estos temas, que los estudias, pones más atención, piensas cómo vas a diseñar los rincones del aula, las actividades que vas a hacer. También cómo puedes tratar el tema en la programación anual, en las reuniones con las familias...». En este sentido, como se ha visto más arriba, las dudas planteadas por las familias y la forma de abordarlas aparecen como otro punto para su formación: «una amiga que es maestra de infantil me contó que tenía un niño que le gustaba mucho ponerse un tutú de bailarina que tenían en clase y un día se le llevó a casa debajo del babi. Al día siguiente el padre apareció en clase asustado porque su hijo se ponía cosas de niña... le preguntaba a la profesora si de mayor no tendría un problema de identidad...».

Además, ofrecen ideas a considerar para su inclusión: «formación práctica, con ejemplos, para saber qué hacer en determinadas situaciones», «una asignatura que te ayude a ver las cosas, a estar atenta». La formación teórica es adecuada, es importante conocer lo que significa la coeducación afirman, aunque es fundamental saber que «si veo que pasa esto en el aula, saber cómo trabajarlo, hacer esta u otra dinámica». Más aun, como afirma una de las estudiantes, «creo que debería trabajarse en el modo de proceder y de hablar y pensar de los maestros, no es ni una charla ni una asignatura, sino un clima que debe conseguirse en el centro». 
Ante determinadas afirmaciones incluidas en el cuestionario relacionadas con la igualdad entre mujeres y hombres y con los diferentes roles a desarrollar en nuestra sociedad por unas y otros, nos encontramos que, si bien hay esferas en las que las diferencias se van diluyendo, en otras continúan manteniéndose. Así, debe destacarse lo siguiente (Tabla 1):

- Las opiniones ante el reparto de las responsabilidades de las tareas domésticas, cuidado de los hijos/as..., muestran una clara orientación hacia la desaparición de tareas tradicionalmente asignadas a mujeres y hombres. Ante la pregunta Hombres y mujeres pueden realizar de la misma manera las tareas domésticas, un $96 \%$ se muestra muy de acuerdo o de acuerdo y sólo un $3 \%$ rechaza la idea.

- En la misma línea, cuando son preguntados por la posibilidad de que si en una pareja (hombre-mujer) uno de sus miembros debe quedarse en casa para ocuparse de sus hijos/as, es mejor que lo haga la mujer, el $98 \%$ no acepta esta alternativa (muy en desacuerdo o en desacuerdo con ella).

- Del mismo modo, en el plano del trabajo extradoméstico, se debe señalar que la mayoría $(83 \%)$ se muestra en contra de la idea de que Hay trabajos que deben ser realizados por hombres y otros por mujeres. Sólo un 7,5\% mantiene esta afirmación.

- Piensan además que mujeres y hombres tienen la misma capacidad para desarrollar puestos directivos. Un $92,5 \%$ se muestra muy en desacuerdo o en desacuerdo con que Los hombres están más capacitados que las mujeres para los puestos directivos.

- Además, el 70\% no está de acuerdo con la idea de que La dirección y el mando son cualidades más habituales en los chicos que en las chicas.

- En aquellas cuestiones relacionadas con el mundo escolar, debe destacarse cómo un 35\% del alumnado se muestra muy de acuerdo o de acuerdo con la idea de que las niñas son más tranquilas en el aula que los niños y un $26 \%$ no sabe posicionarse ante esta afirmación. De otro lado, la mayoría (55\%), se muestra muy en desacuerdo o en desacuerdo con la afirmación Las chicas están mejor preparadas para algunas materias que los chicos. Un $27 \%$ reconoce esta idea.

- Sin embargo, sus opiniones son menos extremas cuando se habla de otras situaciones, dado que como muestran los datos obtenidos, no tienen claro que la sociedad acabe de aceptar formas de ser que hablan de una nueva masculinidad en la que los hombres expresan sus sentimientos. Así, ante la afirmación En nuestra sociedad está bien visto que los hombres muestren sus sentimientos públicamente, un $49 \%$ piensa que es así, si bien un $21 \%$ se muestra muy en desacuerdo o en desacuerdo con la idea, y un 30\% indeciso/a.

- Opinan además que la igualdad sigue sin conseguirse en España: el 72\% del alumnado encuestado se muestra muy en desacuerdo o en desacuerdo con la idea de que la igualdad entre mujeres y hombres se ha logrado. 
Tabla 1

\begin{tabular}{l|c|c|c|c}
\hline \multicolumn{5}{c}{ Grado de acuerdo con las siguientes afirmaciones } \\
\hline $\begin{array}{l}\text { De acuerdo } \\
\text { y muy de } \\
\text { acuerdo } \\
\mathbf{( 5 - 4 ) ( \% )}\end{array}$ & $\begin{array}{c}\text { No lo tengo } \\
\text { claro } \\
\mathbf{( 3 )}(\%)\end{array}$ & $\begin{array}{c}\text { En desacuerdo } \\
\text { y muy en desa- } \\
\text { cuerdo (2-1) (\%) })\end{array}$ & $\begin{array}{c}\text { Media } \\
\text { del } \\
\text { grado }\end{array}$ \\
\hline $\begin{array}{l}\text { Las niñas son más tranquilas en el aula } \\
\text { que los niños }\end{array}$ & 34,7 & 26,5 & 38,8 & 2,89 \\
\hline $\begin{array}{l}\text { Las chicas están mejor preparadas para } \\
\text { lgunas materias que los chicos }\end{array}$ & 26,8 & 15,5 & 57,7 & 2,47 \\
\hline $\begin{array}{l}\text { La dirección y el mando son cualidades } \\
\text { más habituales en los chicos que en las } \\
\text { chicas }\end{array}$ & 15,5 & 14,4 & 70,1 & 2,47 \\
\hline $\begin{array}{l}\text { Los hombres están más capacitados que } \\
\text { las mujeres para los puestos directivos }\end{array}$ & 2,1 & 5,4 & 92,5 & 1,35 \\
\hline $\begin{array}{l}\text { Hay trabajos que deben ser realizados } \\
\text { por hombres y otros por mujeres }\end{array}$ & 7,5 & 9,7 & 82,8 & 1,6 \\
\hline $\begin{array}{l}\text { Hombres y mujeres pueden realizar de la } \\
\text { misma manera las tareas domésticas }\end{array}$ & 95,9 & 1,0 & 3,1 & 4,8 \\
\hline $\begin{array}{l}\text { En nuestra sociedad, está bien visto que } \\
\text { los hombres muestren sus sentimientos } \\
\text { públicamente }\end{array}$ & 21,3 & 29,8 & 48,9 & 2,65 \\
\hline $\begin{array}{l}\text { Si en una pareja (hombre-mujer) uno } \\
\text { de sus miembros debe quedarse en casa } \\
\text { para ocuparse de sus hijos/as, es mejor } \\
\text { que lo haga la mujer }\end{array}$ & 0 & 2,3 & 97,7 & 1,23 \\
\hline $\begin{array}{l}\text { La igualdad entre mujeres y hombres } \\
\text { está conseguida en España }\end{array}$ & 23,6 & 4,5 & & \\
\hline
\end{tabular}

Escala: De 5 - Muy de acuerdo; a 1 - Muy en desacuerdo

El análisis de los discursos del grupo de discusión nos ayuda a comprender algunas de estas ideas: coincidiendo con los datos obtenidos en el cuestionario, y a pesar de reconocer los cambios que se han dado en los últimos años en el papel de hombres y mujeres, consideran que la desigualdad se sigue manifestando en diversas esferas de la realidad social, desde la presencia desigual en puestos de responsabilidad, «en España, apenas hay rectoras, no hemos tenido nunca una presidenta del gobierno... seguimos con ese estereotipo que los cargos de poder son para los hombres...», hasta el mantenimiento de estereotipos y conductas atribuidas a uno y otro género desde la infancia que, no se lo olvide, continúa condicionando la vida adulta: «yo le preguntaba a mi amigo que por qué el niño no podía ir disfrazado de princesa y él decía: calla, calla, ¿cómo va a ir disfrazado de princesa?» Una situación a la que la escuela, según su opinión, también 
contribuye a mantener: «en el colegio de mi sobrino hace poco ha habido una fiesta de disfraces y les mandaron una circular en la que les decían que las niñas podían ir disfrazadas de princesas o de enfermeras y los niños de bomberos o de policía...».

En este sentido, nos interesó indagar en una de las ideas que surge tras el análisis de los datos del cuestionario, esto es, los estereotipos de niños y niñas que parecen mantenerse. Así, una de las estudiantes afirmó que «las niñas están siempre más atentas y se les presta menos atención en clase», opiniones que nos hacen pensar en la necesidad de acabar con estas ideas desde su formación inicial, dado que como han demostrado diversos estudios, estos prejuicios acaban influyendo en las relaciones profesorado-alumnado desde su llegada a la escuela. «Durante las prácticas, en mi clase a los niños se les prestaba más atención, a los que se les ayudaba, porque las niñas eran como más espabiladas, más resueltas a la hora de hacer las cosas», relata una de las alumnas en el grupo de discusión. Otra de las participantes, con experiencia en una escuela infantil, aporta otro ejemplo: «Cuando hice las prácticas lo veía, salíamos al recreo, se caía una niña y allí iba todo el mundo: profesoras, cuidadoras..., mientras que si se caía un niño enseguida le decían, venga, no llores, para arriba, no te estés ahí que no pasa nada...».

\section{Conclusiones}

La necesidad de seguir trabajando desde la escuela, acompañada como no podía ser de otra manera de otros agentes como la familia o los medios de comunicación, en la búsqueda de la igualdad entre mujeres y hombres continúa siendo vital en nuestra sociedad. El discurso de la igualdad como algo conseguido ha hecho caer en una trampa que ha llevado al olvido a algunos programas y planes, una situación acompañada por la priorización de otras demandas, sin duda justificadas, durante la crisis económica en la que nos hemos visto envueltos desde la primera década del Siglo XXI (Lombardo y León 2014). Una circunstancia, además, en la que la ideología de los partidos políticos en el poder ha jugado un papel fundamental en el desmantelamiento o pervivencia de este tipo de políticas, de tal modo que, siguiendo a Paleo y Alonso (2014), la existencia de gobiernos de centro-derecha (tanto a nivel estatal como autonómico) ha sido un factor fundamental para el debilitamiento de aquellas medidas implementadas desde el comienzo de las políticas de género en España, así como un freno para el desarrollo de otras nuevas.

He partido de la idea de que frente a décadas anteriores en las que la coeducación impregnaba una buena parte de las prácticas educativas en los niveles pre-universitarios (Romero Díaz y Abril Morales 2008), en la actualidad se asiste a un relajamiento de estas propuestas que, tal y como se ha comprobado con los datos presentados, apenas son recordadas por el estudiantado que ha cursado estos niveles educativos recientemente. En el caso del alumnado de Magisterio, 
esta situación debe ser abordada desde su llegada a la universidad con el fin de promoverlas a su entrada a las aulas como profesionales de la docencia.

Introducir la perspectiva de género posibilitará entre el alumnado, como afirma Ballarín, "comprender la construcción de realidades sociales y científicas desiguales en función de sexo» $(2013,90)$. En este sentido, bien se puede afirmar que la investigación desarrollada ha servido no sólo para conocer opiniones o actitudes de nuestro alumnado, sino también para generar la reflexión sobre un tema que, como bien se ha afirmado en numerosas ocasiones, necesita de las "gafas moradas» para ser visibilizado. Como cuenta una de las alumnas participante en el grupo de discusión, «Hay muchos gestos sexistas que pasan desapercibidos. Desde que vimos estos temas en la asignatura e hicimos la encuesta me fijo más, igual antes me hubiera dado igual, hubiera pasado desapercibido, pero ahora me fijo en todo».

\section{Referencias bibliográficas}

Anguita, Rocío, y Luis M. Torrego. 2009. «Género, educación y formación del profesorado: retos y posibilidades». Revista Interuniversitaria de Formación del Profesorado 64 (23,1): 17-26. Disponible en https://dialnet.unirioja.es/servlet/articulo?codigo=2956461

Ballarín, Pilar. 2013. «Docencia Universitaria y conocimientos en torno al género. Resistencias, creencias y prejuicios». Cuestiones de género: de la igualdad y la diferencia 8: 89-106. DOI: https://doi.org/10.18002/cg.v0i8.880

Calvo, Adelina, Teresa Susinos, y Marta García Lastra. 2011. «El largo camino hacia la coeducación. Un análisis de las etapas de la educación de las mujeres a partir de tres relatos de vida escolar». Revista de Educación 354: 549-573. Disponible en http://www. revistaeducacion.educacion.es/re354_22.html

Coyner, Sandra. 1991. «Women's studies». NWSA Journal 3 (3): 349-354.

Dickinson, Torry D. 2005. «Strengthening women's studies through applied activism: Theoretical, classroom, regional, and cross-border strategies for participating in change». Women's Studies International Forum 28 (2-3): 115-126. DOI: https://doi.org/10.1016/j. wsif.2005.04.002

Donoso-Vázquez, Trinidad, y Anna Velasco-Martínez. 2013. «¿Por qué una propuesta en formación en perspectiva de género en el ámbito universitario?» Profesorado. Revista de curriculum y formación del profesorado 17 (1): 71-88. Disponible en http://www.ugr. es/ recfpro/rev171ART5.pdf

Fernández García, Emilia, y Joaquín Piedra de la Cuadra. 2010. «Efecto de una formación coeducativa sobre las actitudes hacia la igualdad en el futuro profesorado de Educación Primaria». CCD, Cultura_ciencia_deporte 5 (15), 151-158. DOI: https://doi. org//10.12800/ccd.v5i15.106

Fernández Enguita, Mariano. 2016. La educación en la encrucijada. Madrid: Fundación Santillana.

García Lastra, Marta. 2010a. «La voz de las mujeres en la universidad». RASE. Revista de Asociación de Sociología de la Educación 3 (3): 357-368. Disponible en https://ojs.uv.es/ index.php/RASE/article/view/8717

García Lastra, Marta. 2010b. «Género y sexismo en la escuela». Sociología de la Educación Secundaria, coordinado por Rafael Feito, 123-138. Barcelona: Graò. 
García Lastra, Marta, y Belén Díaz Díaz. 2013. «Equality of Opportunities at Spanish Universities?: Learning from the Experience». Géneros. Multidisciplinary Journal of Gender Studies 2 (3): 255-283. DOI: https://doi.org/10.4471/generos.2013.29

Gil-Flores, José Ignacio (1992). «La metodología de investigación mediante grupos de discusión». Enseñanza \& Teaching: Revista Interuniversitaria de Didáctica 10-11:199-212. Disponible en http://hdl.handle.net/11441/16848

Instituto de la Mujer/RED2 Consultores. 2004. Estudio para conocer la situación actual de la educación para la igualdad en España. Madrid: Instituto de la Mujer.

Lombardo, Emanuela, y Margarita León. 2014. «Políticas de igualdad de género y sociales en España: origen, desarrollo y desmantelamiento en un contexto de crisis económica». Investigaciones Feministas 5: 13-35. DOI: https://doi.org/10.5209/rev_INFE.2014. v5.47986

López-Francés, Inmaculada, María Isabel Viana-Orta, y Beatriz Sánchez-Sánchez. 2016. «La equidad de género en el ámbito universitario: ¿un reto resuelto?» Revista Electrónica Interuniversitaria de Formación del Profesorado 19 (2): 349-361. Disponible en http://revistas.um.es/reifop/article/view/211531 [Consultado el 3 de octubre de 2016].

López Navajas, Ana. 2014. «Análisis de la ausencia de las mujeres en los manuales de la ESO: una genealogía de conocimiento ocultada». Revista de Educación 363: 282-308. DOI: https://doi.org/10.4438/1988-592X-RE-2012-363-188

Megías, Ignacio, y Juan Carlos Ballesteros. 2015. Jóvenes y género. El estado de la cuestión. Madrid: Centro Reina Sofía sobre Adolescencia y Juventud; Fundación de Ayuda contra la Drogadicción (FAD). Disponible en http://adolescenciayjuventud.org/ que-hacemos/monografias-y-estudios/ampliar.php/Id_contenido/73892/[Consultado el 14 de junio de 2016].

Paleo, Natalia, y Alba Alonso. 2014. «¿Es solo cuestión de austeridad? Crisis económica y políticas de género en España». Investigaciones Feministas 5: 36-68.

Rebollo, $\mathrm{M}^{a}$ Ángeles. 2013. «La innovación educativa con perspectiva de género. Retos y desafíos para el profesorado. Editorial». Profesorado. Revista de curriculum y formación del profesorado 17 (3): 3-8. Disponible en ttp://hdl.handle.net/11441/43551

Rebollo M ${ }^{a}$ Ángeles et al. 2011. «Diagnóstico de la cultura de género en educación: actitudes del profesorado hacia la igualdad». Revista de Educación 355: 521-546. Disponible en http://www.revistaeducacion.educacion.es/re355/re355_22.pdf

Romero Díaz, Alfons, y Paco Abril Morales. 2008. «Género y la formación del profesorado en los estudios de Educación Infantil». Revista Electrónica Interuniversitaria de Formación del Profesorado 11 (3): 43-51. Disponible en http://www.aufop.com/aufop/ uploaded_files/articulos/1240850050.pdf

Ruiz Olabuénaga, José Ignacio (2003). Técnicas de triangulación y control de calidad en la investigación socioeducativa. Bilbao: Ediciones Mensajero, Fundación Horreum.

Segura Graíño, Rosario. 2010. «El Instituto de la Mujer y los estudios de género en el contexto del Espacio Europeo de Educación Superior». En Miradas multidisciplinares para un mundo en igualdad: ponencias de la I Reunión Científica sobre Igualdad y Género, coordinado por $\mathrm{M}^{\mathrm{a}}$ Josefina Clavo Sebastián y $\mathrm{M}^{\mathrm{a}}$ Ángeles Goicoechea, 61-76. Logroño: Universidad de la Rioja.

Simón. Elena. 2010. La igualdad también se aprende: cuestión de coeducación. Madrid: Narcea. Suberviola Ovejas, Iratxe, y Ma Ángeles Goicoechea. 2010. «Necesidad de introducir la coeducación en la formación inicial del profesorado». En I Congreso Internacional Reiventar la formación docente, 46-62. Málaga: Universidad de Málaga.

Subirats, Marina. 2016. «De los dispositivos selectivos en la educación: el caso del sexismo». RASE 9 (1): 22-36. Disponible en https://ojs.uv.es/index.php/RASE/article/view/8401 
Verge Mestre, Tània y Cabruja Ubach, Teresa. 2017. La perspective de gènere en docència y recerca a les universitats de la Xarxa Vives. Situació actual i reptes de futur. Xarxa Vives d'Universitats. Disponible en http://vives.org/PU3.pdf [Consultado el 10 de marzo de 2017].

Vizcarra Morales, $\mathrm{M}^{\mathrm{a}}$ Teresa et al. 2015. «La perspectiva de género en los títulos de Grado en la Escuela de Magisterio de Vitoria-Gasteiz». Revista de docencia universitaria 13 (1): 297-318. DOI: https://doi.org/10.4995/redu.2015.6448

Woptipka, Christine Min; Francisco Ramírez, y Capitolina Díaz Martínez. 2007. «Un análisis transnacional del surgimiento e institucionalización de los planes académicos de los Estudios de Mujeres». Revista Española de Investigaciones Sociológicas 117: 35-59. Disponible en http://www.reis.cis.es/REIS/PDF/REIS_117_041181905409312.pdf

\section{Referencias legislativas}

Ley Orgánica 1/2004, de 28 de diciembre, de Medidas de Protección Integral contra la Violencia de Género. Disponible en https://www.boe.es/buscar/doc.php?id=BOE-A-2004-21760

Ley Orgánica 2/2006, de 3 de mayo, de Educación. Disponible en http://www.boe.es/buscar/ doc.php?id=BOE-A-2006-7899

Ley Orgánica 3/2007, de 22 de marzo, para la Igualdad efectiva de hombres y mujeres. Disponible en http://www.boe.es/buscar/doc.php?id=BOE-A-2007-6115

Ley 14/2011, de 1 de junio, de la Ciencia, la Tecnología y la Innovación. Disponible en http:// www.boe.es/buscar/doc.php?id=BOE-A-2011-9617

Marta García Lastra es Doctora en Sociología por la Universidad de Deusto. Profesora en la Facultad de Educación de la Universidad de Cantabria, centro en el que actualmente es Vicedecana de Postgrado, donde imparte docencia en diversas asignaturas relacionadas con la Sociología de la Educación y la Sociología del Género en títulos de Grado y Máster. Dirige el Aula Interdisciplinar Isabel Torres de Estudios de las Mujeres y del Género de esta misma universidad. Correo electrónico: garciama@unican.es

Artículo recibido el 29 de mayo de 2017 y aceptado para su publicación el 08 de octubre de 2017. 
